\title{
Structure of Multiwall Nanotubes-Different Models and Electron Diffraction Results
}

\author{
J.M. Zuo, A. Celik-Aktas and M. Gao ${ }^{1}$
}

Department of Materials Science and Engineering, Ferdrick Seitz Materials Research Laboratory, University of Illinois at Urbana-Champaign, Urbana, IL, 61801

Different forms of nanotubes, including single- and multi-wall carbon and boron nitride nanotubes, have received considerable interest due to their unique structure and associated properties. While the structure of a single wall nanotubes (SWNT) nanotube is characterized by its diameter and chiral angle, the structures of multiwall nanotubes (MWNT) are more complicated. Experimentally, a SWNT can be characterized by a variety of techniques, including scanning probe microscopy. Characterization of MWNTs requires a penetrating probe and differentiation of the structure of different walls, which is more challenging. Consequently, our knowledge of MWNT structures are limited compared to SWNTs. The existing structure models for MWNTs include the concentric cylindrical tube model, faceted tubes and tubes made out of a single sheet, or the paper-scroll model. The structure of each walls in a MWNT can be correlated, independent or semi-correlated and the spacing of walls can be random, regular and in between.

We have investigated the structure of multiwall nanotubes using Nanoarea Electron Diffraction (NED), including both carbon double- and multi-wall nanotubes (C-MWNT) and boron nitride multiwall nanotubes (BN-MWNT) [2,3]. Figure 1 compares the electron diffraction patterns recorded from a BN-MWNT and C-MWNT. The two patterns are different. In nanotube diffraction, each wall of a MWNT contributes a set of diffraction spots to the pattern (see Fig. 1c). In case of BN-MWNT, the diffraction spots are grouped and resembles a single tube diffraction. While such grouping is common for BN-MWNT, it is rare for C-MWNT.

Electron images of BN-MWNT show zig-zag like contrast (Fig. 2), which is not in carbon nanotubes such as the one shown in Fig. 1b. To clarify the structure of BN-MWNT, we recorded a series of electron diffraction patterns from different sections of the tube using a nanometersized electron probe. An example is shown in Fig.2. Interpretation of the diffraction patterns revealed that the tube consists of two helices, one highly crystalline and faceted and the other circular and less ordered. The structure is present in BN tubes with similar chirality among its different walls [4].

[1] M. Gao, Current Address, Department of Electrical and Computer Engineering, Ohio State University, Columbus, OH 43210

[2] A.C. Aktas, J.M. Zuo, J.F. Stubbins, C. Tang and Y. Bando, "Chirality Distribution in Boron Nitride Nanotubes", Appl. Phys. Lett., 86, 133110, (2005)

[3] A.C. Aktas, J.M. Zuo, J.F. Stubbins, C. Tang and Y. Bando, "Double-helix Structure in Multiwall Boron Nitride Nanotubes", Acta Cryst A, , (2005)

[4] This work was supported by DOE BES DEFG02-01ER45923 and DEFG02-91ER4539 

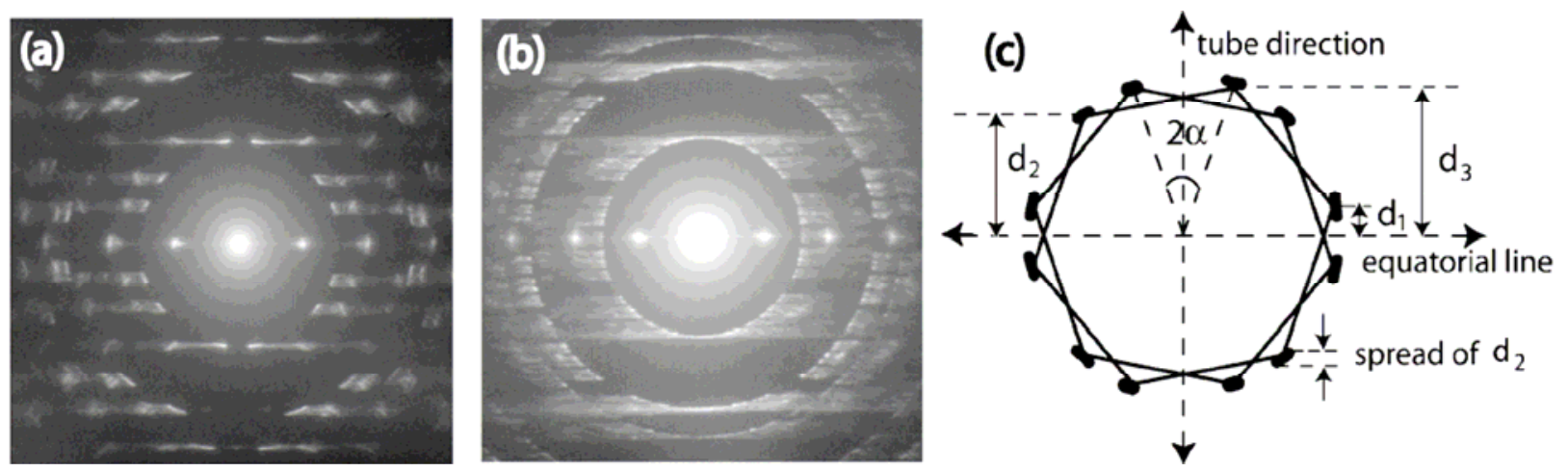

Fig. 1 Electron diffraction patterns recorded from two different multiwall nanotubes a) Boron Nitride, b) carbon and c) schematic diffraction pattern from a single wall nanotube.
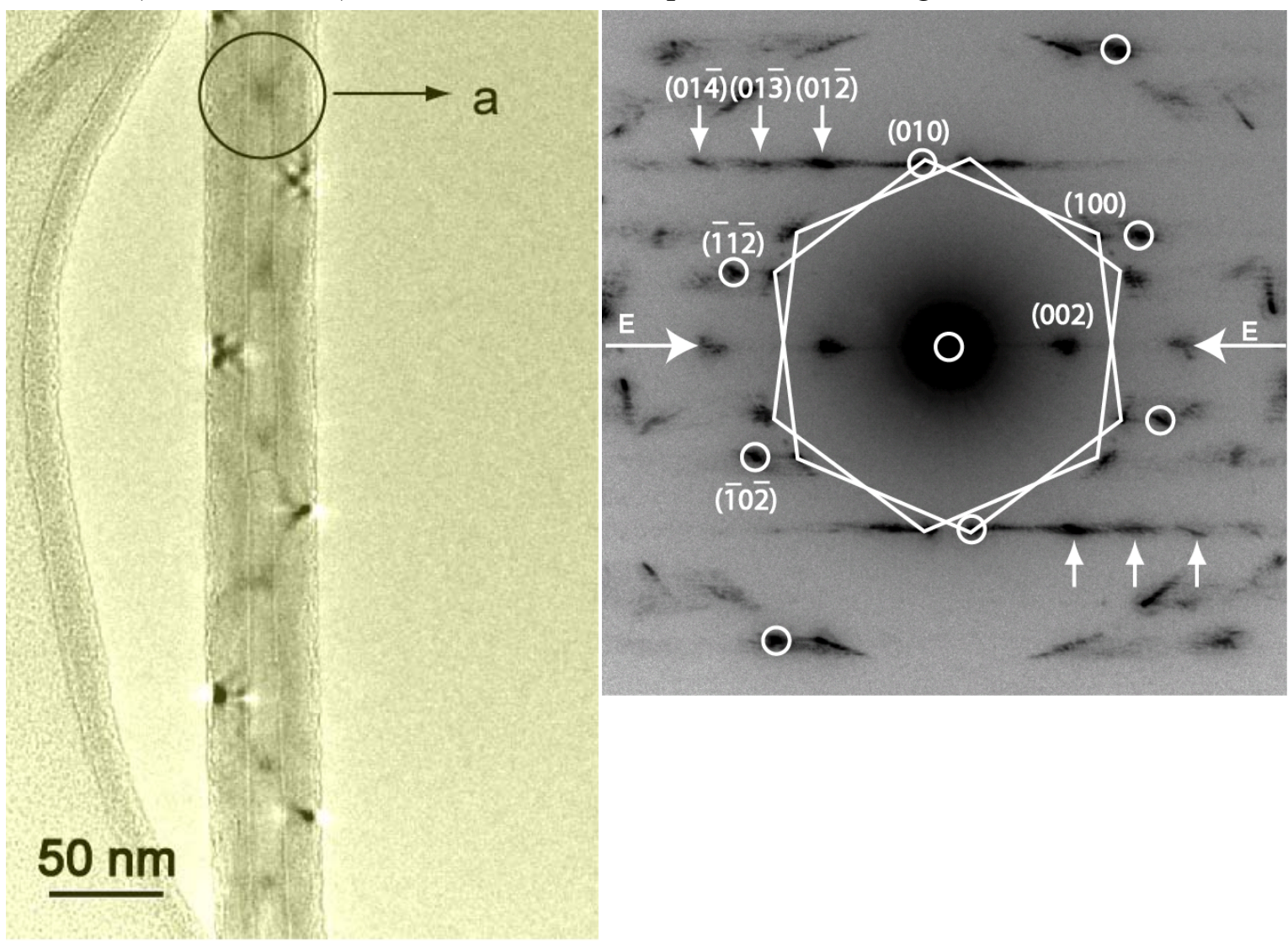

Fig. 2 right) Electron image of a BN-MWNT showing the zig-zag structure. left) The diffraction pattern recorded from the circled area of the tube. 